\title{
3D Rekonstruktion der Magnetfeldvektoren Bx, By, Bz in Cern, Schweiz. 3D Single Chip Hall Sensor 5 Tesla, PHE Fehler 20 ppm.
}

\author{
Pieter Hoeben \\ Hoeben Electronics, Ronkert 44, 5094EW Lage Mierde, Niederlande \\ Tel: +31 135096200, E-Mail: info@hoeben.com
}

Die genaue Messung von starken Magnetfeldern ist heute zum Beispiel mit NMR Messgeräten möglich. NMR Messergebnisse sind jedoch mit dem Nachteil behaftet, dass nur die Stärke des Magnetfeldes gemessen wird, nicht jedoch die Richtung des Magnetfeldes.

Hall Sensoren ermöglichen die Messung sowohl der Stärke des Magnetfeldes als auch dessen Richtung.

Dies ermöglicht einen völlig neuen Lösungsansatz in Cern (Europäische Organisation für Kernforschung), durch den Einsatz des neu entwickelten dreidimensionalen, würfelförmigen Hall Sensor Modules HE444.

Der Einsatz des 3D Hall Sensor Modules ermöglicht die Messung von allen sechs relevanten Messgrössen, sowohl die Feldstärken, als auch die Richtungsvektoren Bx, By und Bz.

Cern hat in der Vergangenheit 3D Sensoren auf Basis der 1D Siemens Hall Sensoren KSY44 gebaut.

Dabei wurden die 1D Sensoren KSY44 als quasi 3D Modell durch das nachfolgend beschriebene 3 D Modell abgebildet, (siehe hierzu Bild 2, Bild 3 und Bild 4).

Da die Anwendung (3D Rekonstruktion der Vektoren Bx, By und Bz) höchste Präzision erfordert, müssen alle drei Achsen bzw. die implementierten Sensoren bezüglich der Empfindlichkeit, des Temperaturverhaltens (mit Bezug auf Nullpunkt, Offset), nahezu identisch sein. Kleine Unterschiede der Achsen zum Beispiel verursacht durch unterschiedliche Empfindlichkeit oder Temperaturverhältnisse der Sensoren, führen zu Messfehlern bezüglich der Richtung der Magnetfelder. Die Orthogonalität der Achsen, hat ebenso wie die Unabhängigkeit der drei Achsen höchste Priorität. 


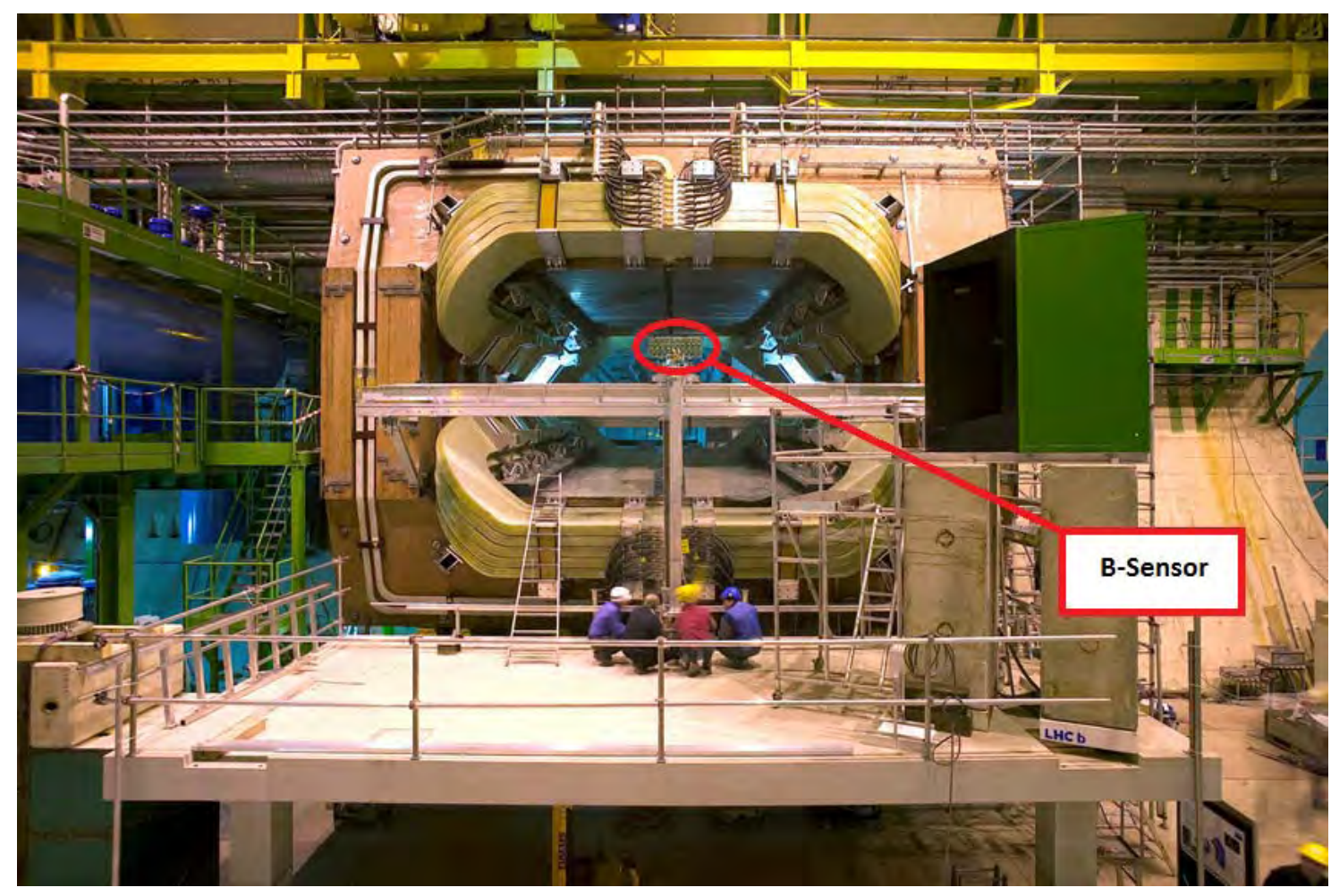

Bild 1: Ein Beispiel für Magnetfeldmessungen in Cern, "Large Hadron Collider"

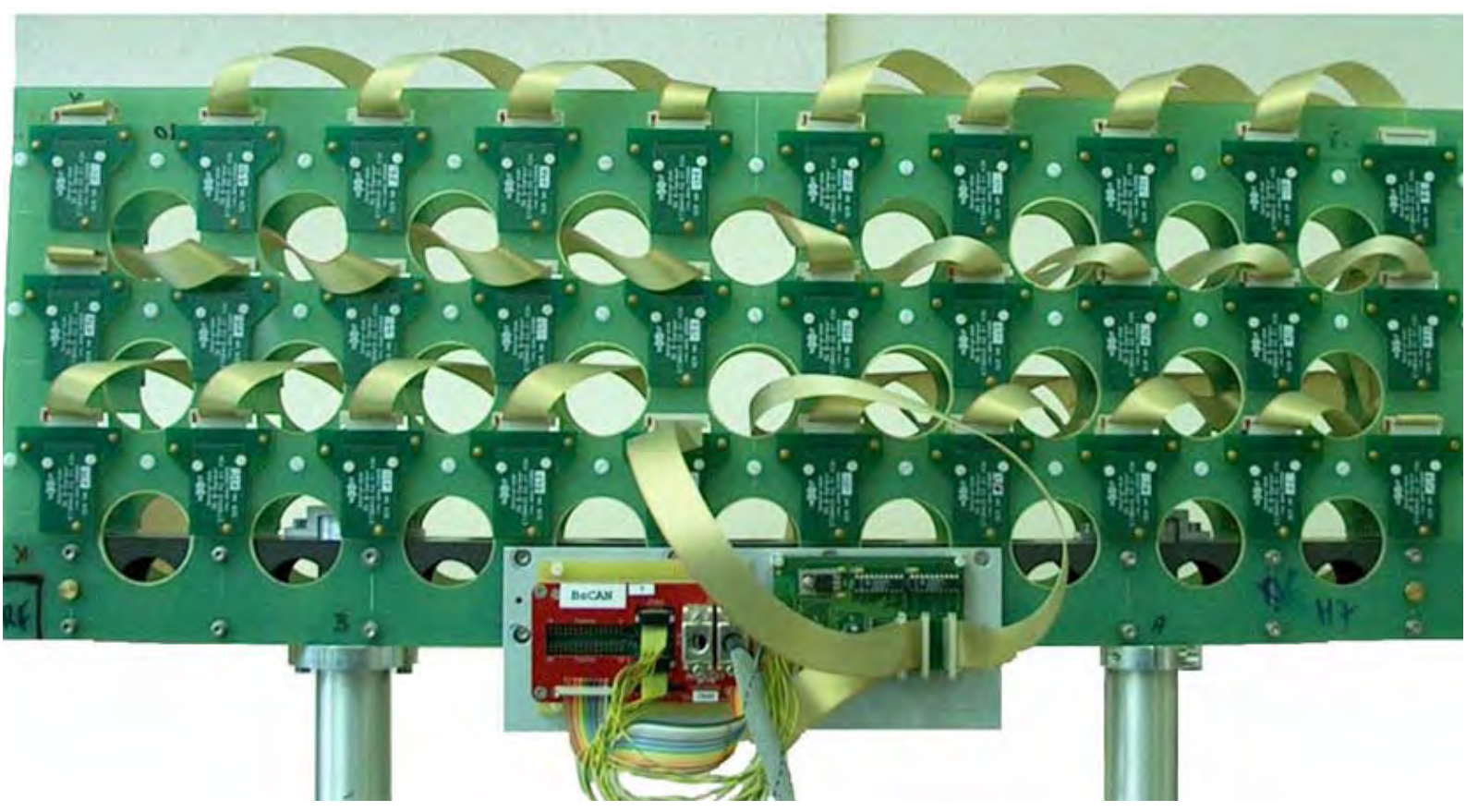

Bild 2: Das Magnetfeld wird mit mehreren 3D Magnetfeldmodulen gemessen, jedes dieser einzelnen Module (hier im Beispiel sind das 30 Mess-Stellen) enthält einen 3D Hall Sensor, basierend auf einem 3D Modell, das aus drei Siemens KSY44 aufgebaut ist. 




Bild 3: Zeigt ein Modul, jedes einzelne Modul hat drei KSY44 Hall Sensoren, die als 3D Hall Sensor Modell angeordnet sind.

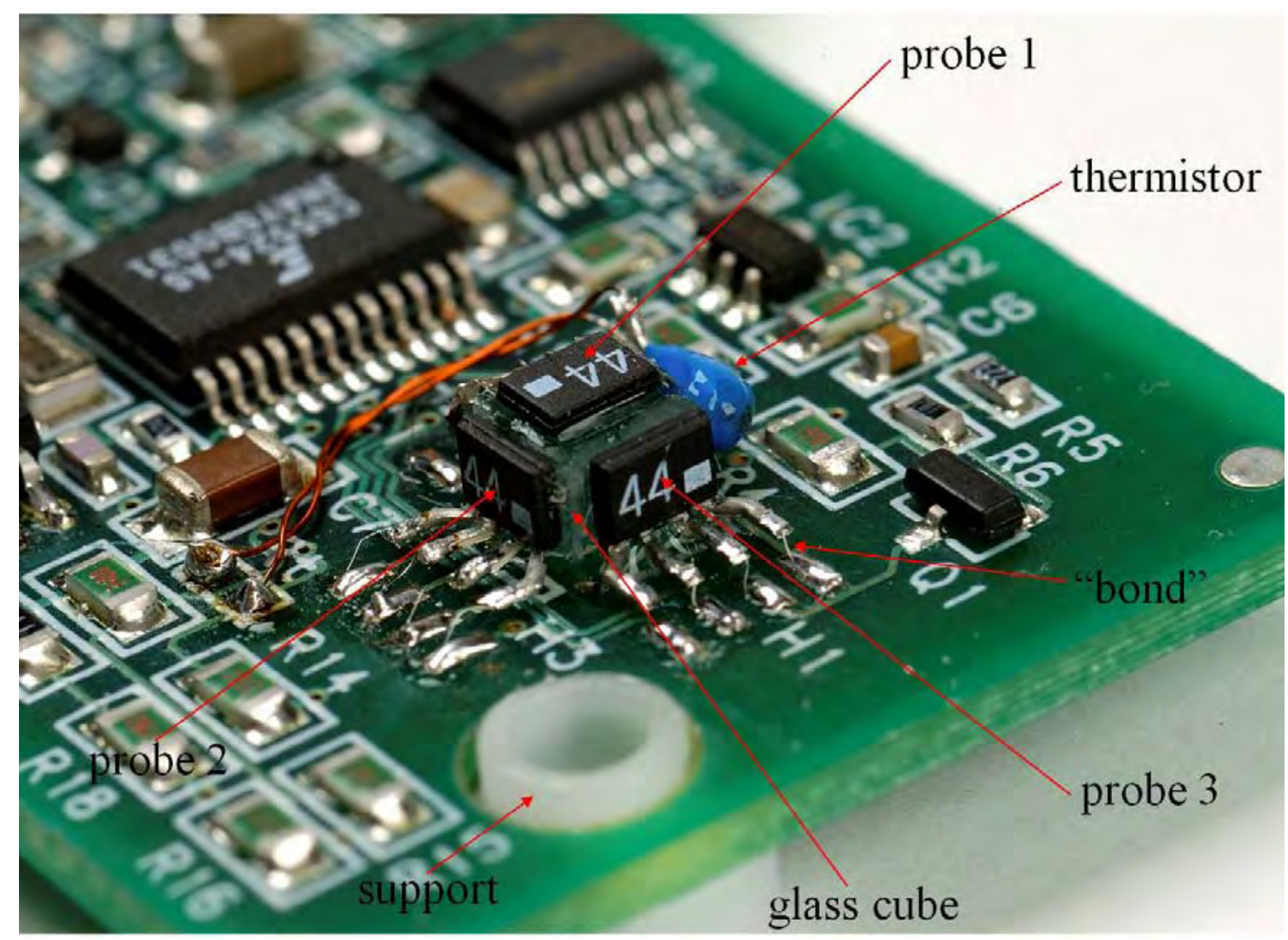

Bild 4: Zeigt einen Detailauschnitt der Nachbildung des Cern 3D Hall Sensors auf dem Modul durch 3 Siemens KSY44 Hall Sensoren. Probe 1, Probe 2 und Probe 3 zeigen die einzelnen 1D Hall Sensoren KSY44. Hier erkennt man deutlich die würfelförmige Nachbildung der drei Achsen durch die drei diskreten Siemens Hall Sensoren KSY44. Dieser, bisher bei Cern mit diskreten Hall Sensoren realisierte Aufbau kann jetzt durch die neu entwickelte Single-Chip 3 D Hall Sensor "HE444" Lösung von Hoeben Electronics mit einem um den Faktor 500 reduzierten PHE Fehler (siehe hierzu Bild 10) realisiert werden. 


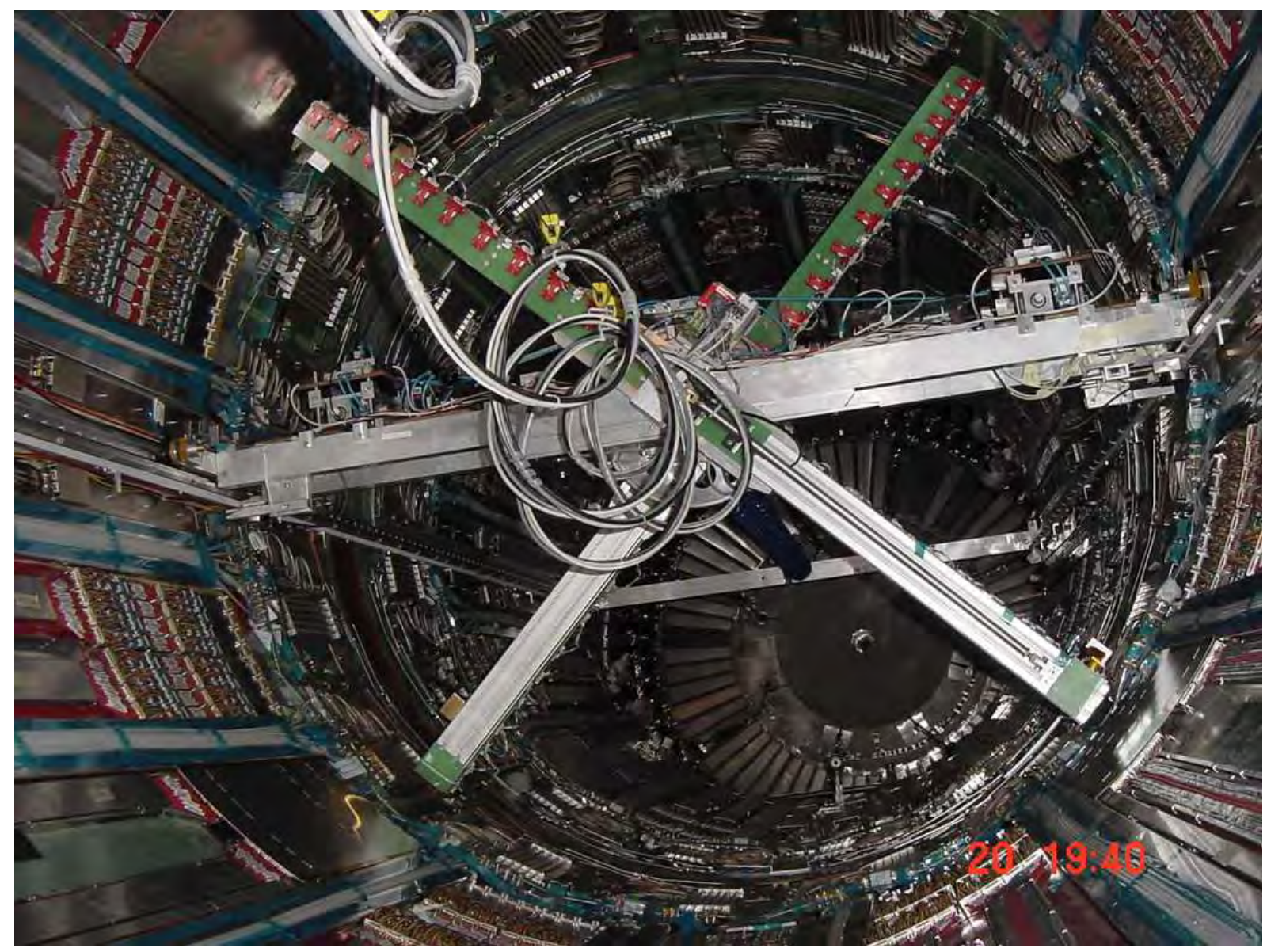

Bild 5: Diese Module werden auch für die CERN Atlas Detektoren benutzt.

ATLAS ist ein Teilchendetektor am europäischem Kernforschungszentrum Cern. ATLAS soll das Higgs-Boson Teilchen nachweisen. Es hat ein starkes, genaues Magnetfeld von 2 Tesla, welches geladene Teilchen zum Detektor ablenkt. Der B-Sensor ist der 3D Hall Sensor von Cern und wird benutzt um dieses Magnetfeld zu messen.

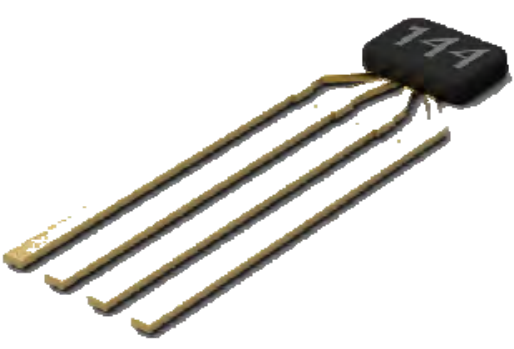

Bild 6: HE144 Hall Sensor

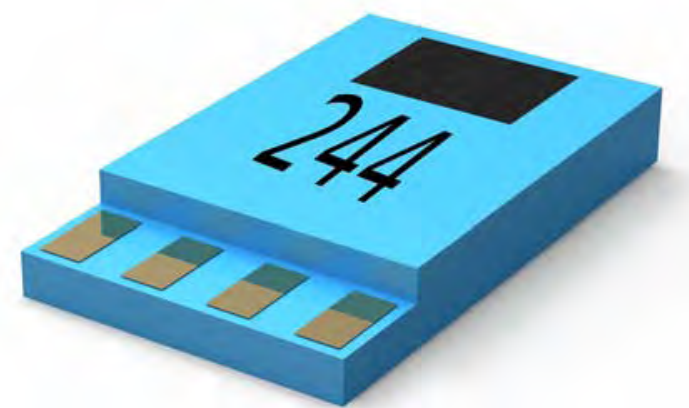

Bild 7: HE244 Hall Sensor

Zum Einsatz im 3 D Hall Sensor HE444 kann man prinzipiell die DIE`s der 1D Hall Sensoren HE144, oder auch die DIE`s des 1D Hall Sensors HE244 verwenden. Für High End Anwendungen mit höchster Genauigkeit, so beim Beispiel Cern gefordert, kommen ausschliesslich die DIE`s des HE244 beim 3D Hall Sensor Hybridbaustein HE444 zum Einsatz. 
Hoeben Electronics hat vor kurzem den 3D Hall Sensor HE444 entwickelt.

In dem 3D Hall Sensor HE444 wird der schon länger am Markt verfügbare 1D Hall Sensor HE244 dreimal eingesetzt. Der auf Keramikbasis bestehende 3D Hall Sensor wurde für Anwendungen, wie die in diesem Vortrag beschriebene Anwendung bei Cern entwickelt.

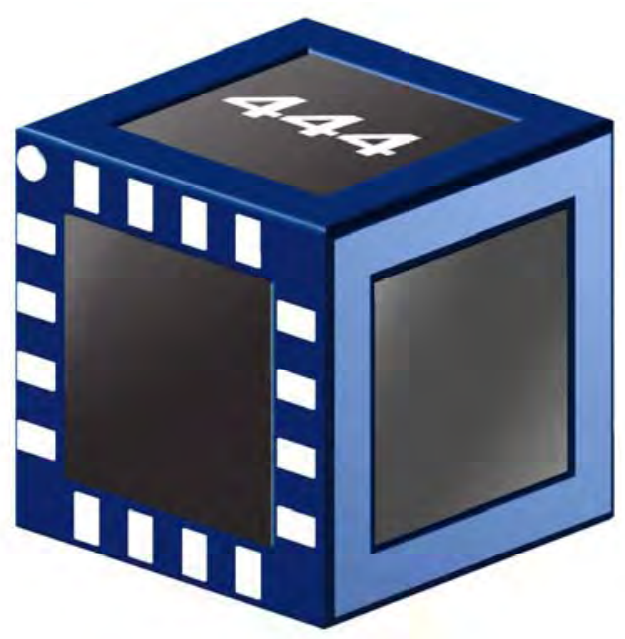

Bild 8: 3D Hall Sensor HE444

\section{Konstruktive Überlegungen}

Manche Anwender wollen in einem bestimmten, beliebig wählbaren Punkt ein Magnetfeld und dessen Richtung messen, dann bedeutet dies, dass sich alle drei Achsen zueinander kreuzen. Drei Hall Sensoren an einem Punkt zu montieren funktioniert jedoch in der Praxis nicht, da alle Sensoren sonst direkt auf den Achsen stehen würden, nicht aber wir gefordert im Mittelpunkt der Achsen. Die Achsen müssen sich in ein Punkt kreuzen. Deswegen wurde bei dem 3D Hall Sensor Modul HE444 eine würfelförmige Bauform gewählt. 


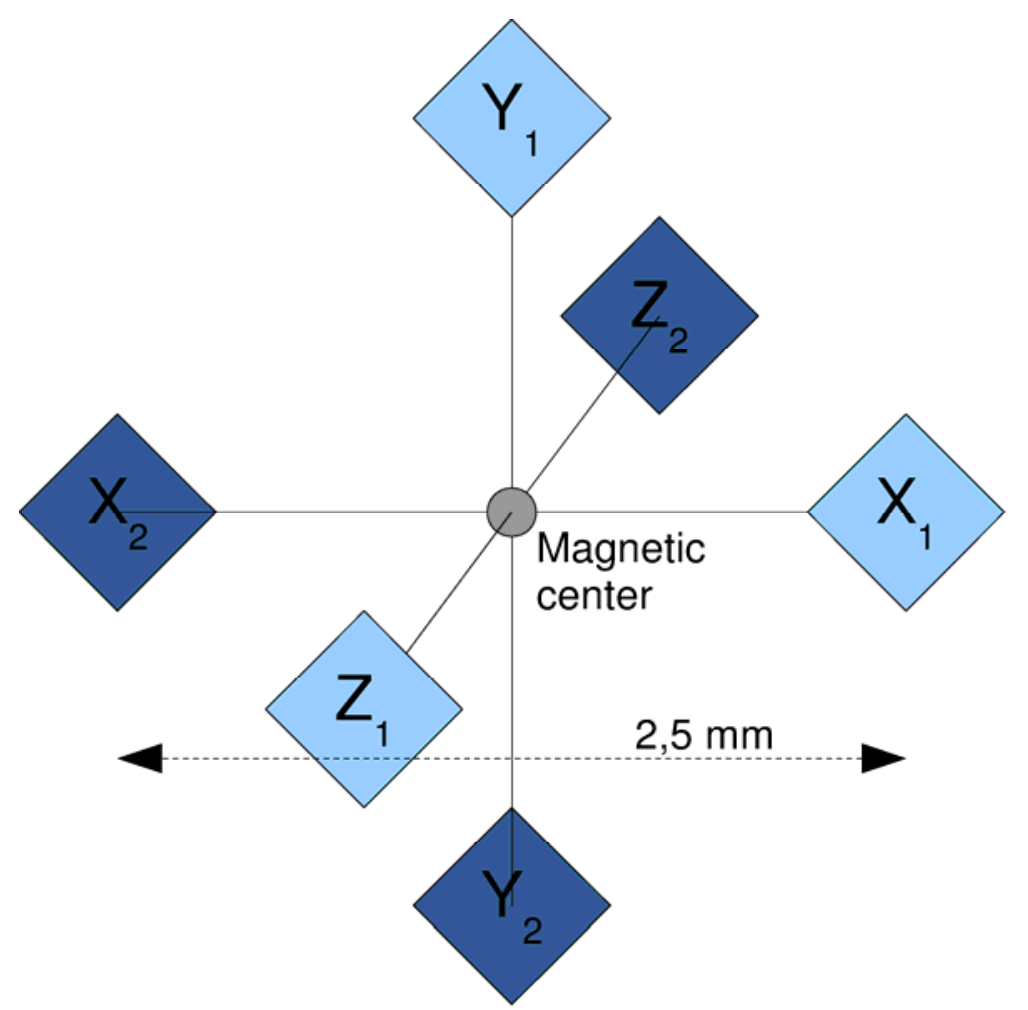

Bild 9: Magnetisches Zentrum vom Hall Sensor

\section{Konstruktiver Aufbau des 3D Hall Sensor Modules HE444:}

Um mit einer sehr hohen Genauigkeit messen zu können, werden zwei Sensoren für jede Achse benutzt, von denen jeder Sensor den gleichen Abstand zum Mittelpunkt hat. Durch Auswertungen vom Mittelwert des Ausgangs der Sensoren kann man sehr genau den Wert vom Magnetfeld im Mittelpunkt zwischen den Sensoren messen. Jede Achse ist in derselben Art realisiert, das sind also 3 Achsen $\times 2$ Sensoren $=6$ Sensoren .

Kunden wie Cern brauchen die genaue Position des Messpunktes. In einem homogenen Magnetfeld ist der Messpunkt immer genau in der Mitte, egal ob drei oder sechs Sensoren benutzt werden. Man kann immer die Größe und die Richtung des Magnetfeldes messen.

In einem nicht-homogenes Magnetfeld jedoch, hat man mit drei Sensoren, nur X1, Y1 und Z1 (siehe Bild 9) eine Verschiebung vom Zentrum. Wenn zum Beispiel ein Magnet nahe am X1 Sensor angeordnet ist, dann sieht dieser Sensor ein starkes Magnetfeld.

Wenn aber derselbe Magnet nahe an der Position ist, wo der X2 Sensor sein sollte, dann sieht der Sensor X1 nur wenig Magnetfeld. Es gibt also eine Verschiebung vom Zentrum in die Richtung des Sensors X1.

Wir haben dieses Problem durch die Verwendung von sechs Sensoren gelöst. Wenn jetzt ein sehr starkes nicht-homogenes Magnetfeld beim Sensor X2 vorhanden ist, dann sieht dieser Sensor ein starkes Magnetfeld, mehr als da wirklich im Mittelpunkt vorhanden ist.

Der Sensor X1 sieht ein viel kleineres Magnetfeld. Wir messen den Mittelwert. Das heißt, dass das gemessene Feld wirklich dem real vorhandenen Feld im Mittelpunkt entspricht. Die Restfehler, die noch vorhanden sind, sind sehr klein und unbedeutend. 


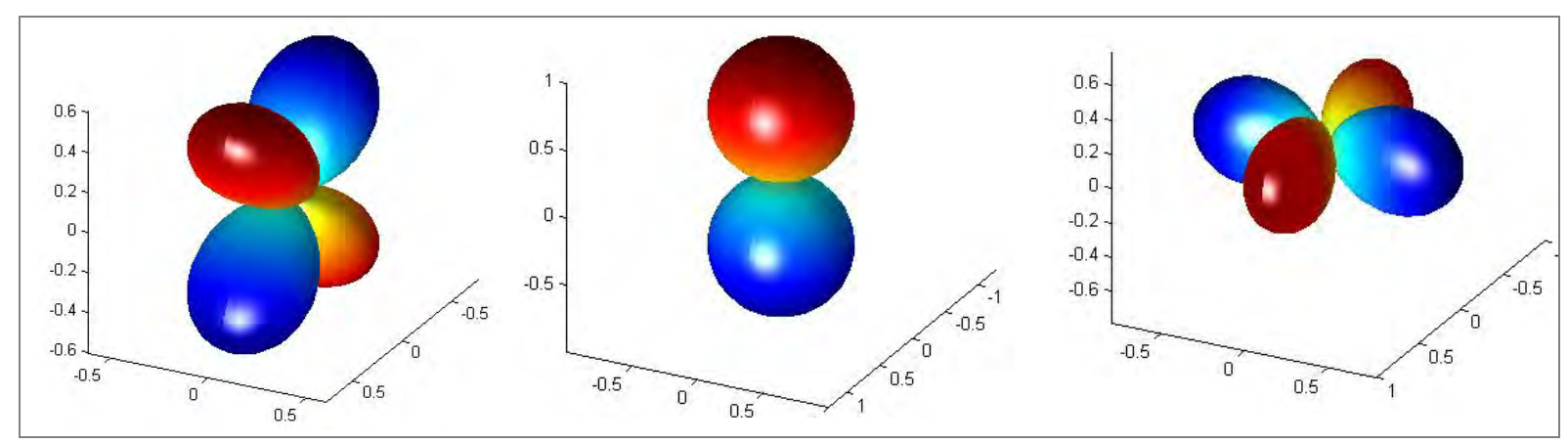

Bild 10: Diagramme Planar Hall Effekt

\section{PHE Fehler ( Planar Hall Effect Fehler)}

Die Sensoren, oder besser die Achsen, dürfen sich gegenseitig nicht beeinflussen. Eine Kenngröße für die Unabhängigkeit der Achsen ist der sogenannte PHE Fehler (Planar Hall Effect Fehler).

1D Hall Sensoren sollten idealerweise nur das Magnetfeld in Z-Richtung zeigen. Der PHE Fehler erzeugt aber auch eine Ausgangsspannung bei Magnetfelder in $X$ - und Y-Richtung. Noch gravierender ist, das sich meist der größte Fehler als $X$ - mal Y-Multiplikation zeigt.

Die aktuell am Markt verfügbaren Hall Sensoren zeigen Schwächen bei der Unabhängigkeit der Achsen, der PHE Fehler liegt bei ca. 1\%. Dieser Fehler erzeugt bei 3D Messungen viele Schwierigkeiten beim Kalibrieren und Messen.

Die Achsen sind dadurch nicht mehr voneinander unabhängig, und beeinflussen sich gegenseitig.

Für jede Messung von einer Achse, müssen auch alle anderen Achsen gemessen werden, um die durch den großen PHE erzeugten Fehler zu beseitigen. Wobei die andere Achse wieder durch die erste Achse beeinflusst wird.

Alle Hoeben Electronics Sensoren haben einen sehr viel niedrigeren PHE.

Der 3D Hall Sensor HE444 basiert auf den Hall Sensoren HE144 oder HE244 mit diesem niedrigen PHE. Der Sensor hat durch spezielle konstruktive Maßnahmen einen um ca. den Faktor 500 reduzierten PHE Fehler, im Vergleich zu den von Cern bisher verwendeten Siemens KSY44 Hall Sensoren. Zum Beispiel nur 20 PPM (20 part per million) pro Achse. Der PHE Fehler des KSY44 liegt bei ca. $1 \%$, der des HE444 bei 0,002\%. Die PHE Fehler Differenz liegt also im Vergleich zum KSY44 von Siemens bei dem Faktor 500. Dieser Faktor behält ebenso seine Gültigkeit im Vergleich mit den bisher aktuell am Markt verfügbaren 3D Hall Sensoren unserer Konkurrenten.

\section{Voraussetzungen}

Folgende Voraussetzungen pro Achse müssen für einen guten 3D Hall Sensor erfüllt sein:

1. Die Sensoren müssen sehr genau sein.

2. Alle Achsen (Sensoren) müssen gleichartig sein.

3. Die Sensoren dürfen nur einen sehr niedrigen PHE Fehler ausweisen. 


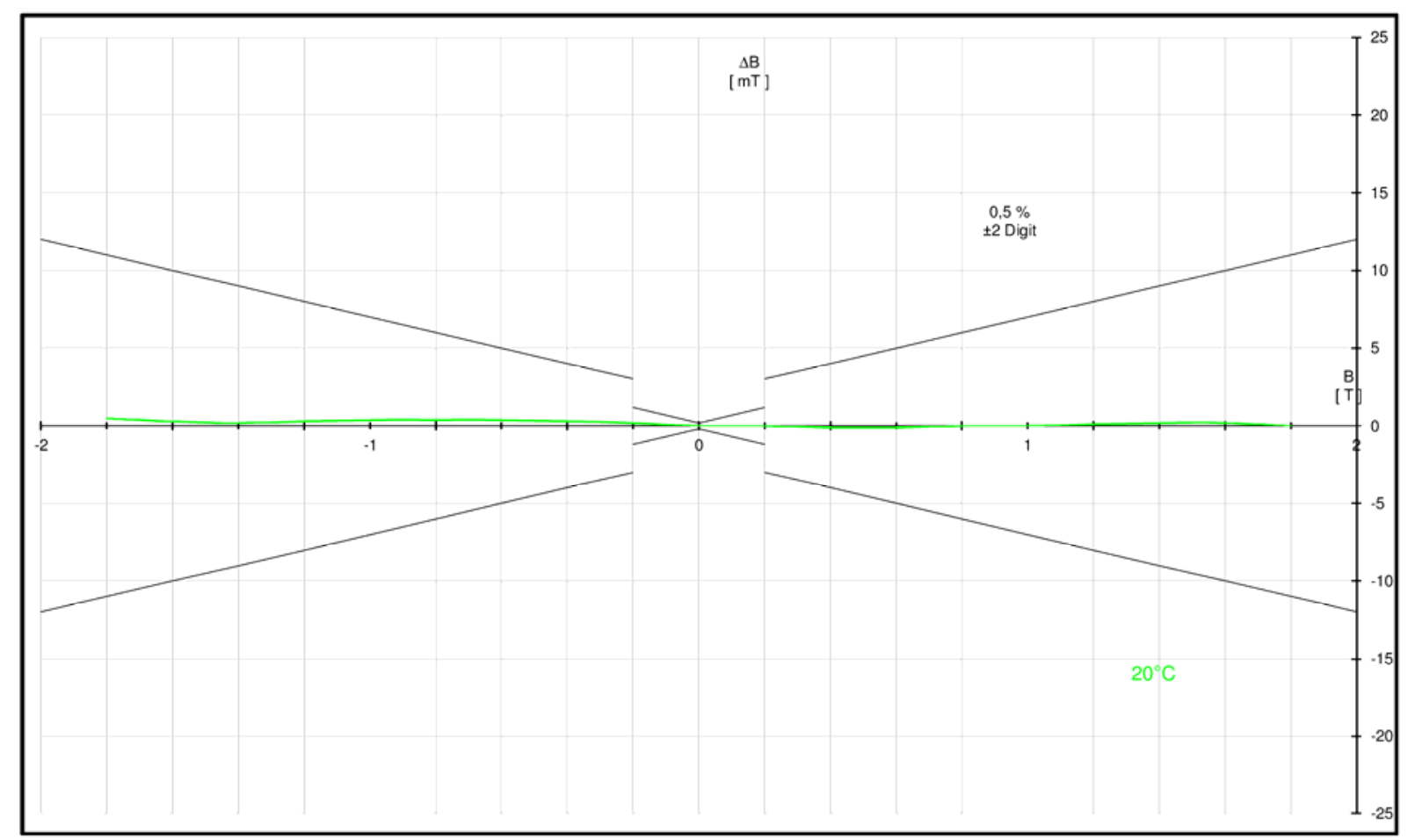

Bild 11: Linearitätsfehler des HE144 Hall Sensors

Hoeben Electronics hat mit der HE444 einen 3D Hall Sensor entwickelt, der auf den Hoeben Electronics Hall Sensoren HE144 und HE244 basiert. Die erste Voraussetzung ist damit erfüllt: Diese Sensoren erreichen eine sehr gute Linearität (unkalibriert schon $0,1 \%$ ) und ein gutes Temperaturverhältnis. Auch die zweite Voraussetzung ist erfüllt, die Sensoren sind einander sehr ähnlich, dies macht Anwendungen mit mehreren Sensoren viel einfacher.

Die Sensoren können auch sehr starke Magnetfelder messen (5 Tesla, und sogar viel mehr, sind möglich). Da das Rauschen und die Drift minimal sind, ist es möglich, auch sehr kleine Magnetfelder zu messen. Der größte Vorteil des Einsatzes von HE144 und HE244 ist aber der geringe PHE Fehler. So lag dieser Fehler, abhängig von der Richtung bei den abgekündigten Siemens KSY44 Sensoren, bei etwa 0,5 bis 1\%. Bei den Hoeben Electronics Typen HE144 und HE244 ist der PHE Fehler schon viel niedriger. Beim 3D Hall Sensor werden PHE Werte von 20 ppm $=0,002 \%$ erreicht.

Das Gehäuse des HE444 basiert auf Keramik. Dadurch besteht die Möglichkeit einen sehr großen Temperaturbereich von nur einigen Kelvin bis weit über $250{ }^{\circ} \mathrm{C}$ abzudecken. In allen sechs Würfelflächen ist jeweils ein Hall Sensor angeordnet.

Die Abmessungen des würfelförmigen Sensors sind $3.3 \times 3.3 \times 3.3 \mathrm{~mm}$. An der Unterseite vom HE444 sind alle drei Achsen separat verfügbar (siehe Bild 8). Elektrische Daten und Auswertungen vom HE444 können den Datenblättern des HE144 und HE244 entnommen werden. Dies erleichtert die Elektronik-Entwicklung, da man mit einem PIN-Bauteil pro Achse arbeiten kann.

Das Gehäuse und die verwendeten Materialien vom HE444 sind für die Nutzung auf Platinen, Keramik etc., mit einen niedrigem Temperaturkoeffizienten optimiert. Das Bauteil kann gelötet oder bei Bedarf auch gebondet werden. 


\section{Hoeben Electronics Hall Sensoren}

Ab Lager sind HE144 und HE244 Sensoren verfügbar.


Bild 12: HE144 und HE244S Hall Sensoren

Die Twisted Pair Version, HE244T, gibt ein sehr gute Störunterdrückung, eine hohe Bandbreite min. $100 \mathrm{kHz}$ und eine gute mechanische Stabilität. Die Twisted Pairs (Verdrehte Leitungen) sind $20 \mathrm{~cm}$ lang,

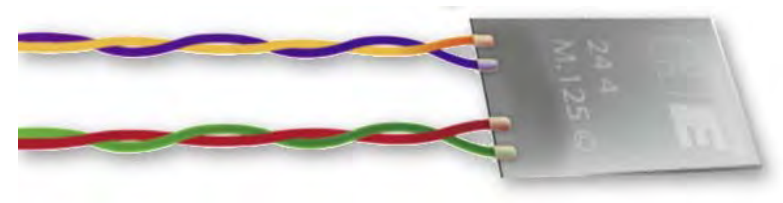

Bild 13: HE244T Hall Sensor

Auf Kundenwunsch sind sämtliche denkbaren kundenspezifischen Bauformen möglich.
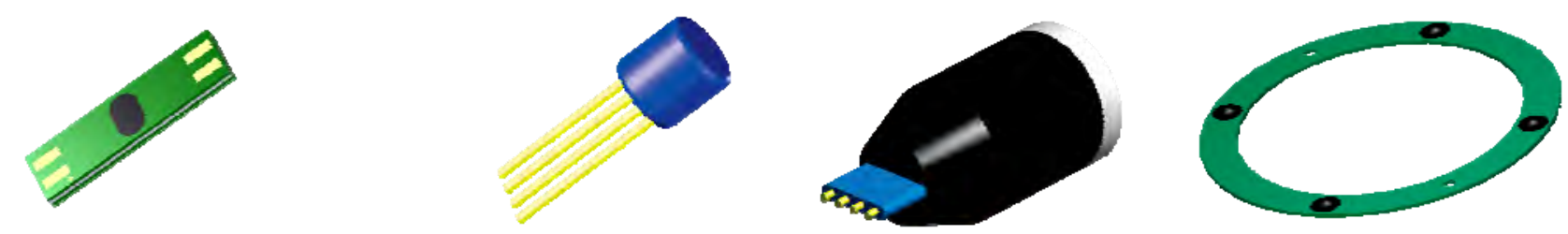

Bild 12: Spezielle Bauformen

Ebenso ist die Integration in Flex Foil möglich.

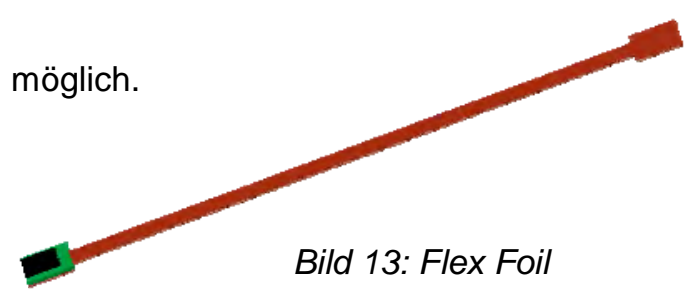

Die Hoeben Electronics sind Hall Sensoren ohne eingebaute Elektronik. Alle Achsen müssen mit einem Konstantstrom gespeist werden. Abhängig vom Messbereich empfehlen wir normalerweise ca. $1 \mathrm{~mA}$. Am Ausgang muss man hochohmig auslesen. 


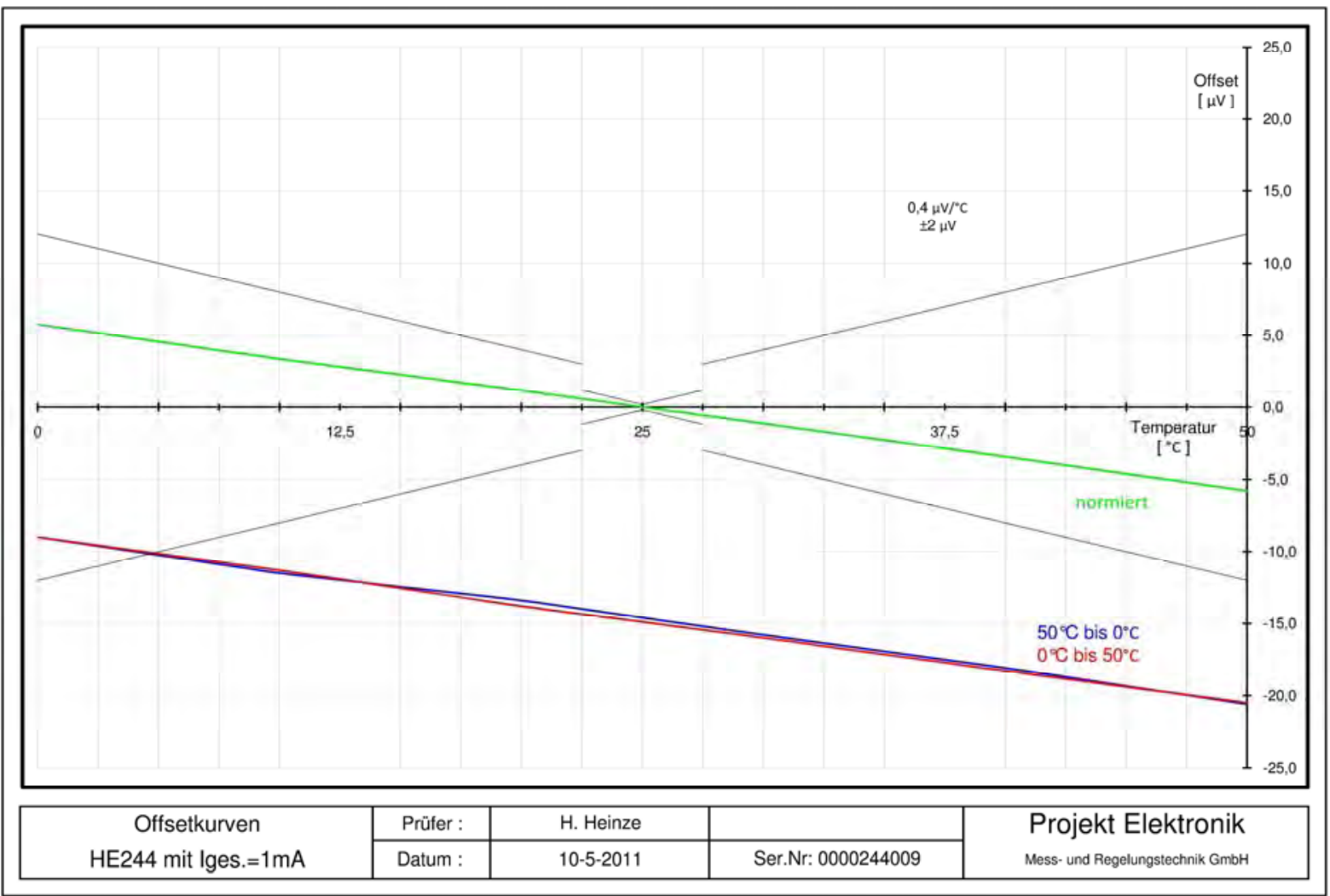

Bild 12: Nullpunktdrift eines 1D Hall Sensors HE244

\section{Zusammenfassend}

3D Hall Sensoren, welche auf quasi zwei dimensionalen IC-Bauformen beruhen, (dies trifft auf sämtliche Standard SMD ICs zu) haben sicher im Consumerbereich bei Low End Anforderungen an die Achsenunabhängigkeit ihre Berechtigung.

Es ist jedoch nahezu unmöglich 6 Freiheitsgrade (Feldstärken, Richtungsvektoren) auf der Chip Ebene rechentechnisch genau zu korrigieren.

Will man jedoch neue Anwendungen im Bereich High-End Applikationen (NMR, Qualitätssicherung, Messtechnik) erschließen, dann bleibt nur der Weg zur realen 3D-Bauform (z.B. Würfel Sensor Modul).

Nur das ermöglicht die exakte Messung aller 6 relevanten Freiheitsgrade, sowohl die Feldstärken, als auch die Richtungsvektoren Bx, By und Bz. Diese aktuell rein hardwarebasierende Lösung ermöglicht den direkten Zugriff auf alle 3 Achsen bei einem PHE-Fehler von 0,002\%.

\section{CERN Bilder: ATLAS Experiment $\odot 2011$ CERN}

